\title{
Urorectal Septum Malformation Sequence Associated with Mayer- Rokitansky-Küster-Hauser Syndrome and Ovarian Dysgenesis: An Autopsy Case Report
}

\author{
Darouich S1,2*, Boujelbène $\mathbf{N}^{2,3}$, Mrad $\mathrm{K}^{2,3}$ and Masmoudi $\mathrm{A}^{2,4}$ \\ ${ }^{1}$ Fetopathology Unit, University Hospital Habib Bougatfa, Bizerte, Tunisia \\ 2Faculty of Medicine of Tunis, University of Tunis El Manar, Tunis, Tunisia \\ 3Department of Pathology, Salah Azaiez Institute, Tunis, Tunisia \\ ${ }^{4}$ Department of Embryo-Fetopathology, Maternity and Neonatology Center, \\ Tunis, Tunisia
}

*Corresponding author: Sihem Darouich, Fetopathology Unit, University Hospital Habib Bougatfa, Tunisia, Tel: +216 72110200, Fax: +216 72443500; E-mail: sihem.darouich@free.fr

\section{Abstract}

Objectives: The urorectal septum malformation sequence (URSMS) results from failure of cloacal division by the urorectal septum. It may be associated with genital and gonadal malformations which are related to defects of the other mesodermal-derived structures, Müllerian ducts and genital crests. In this report, we aim to describe this malformative association not previously reported in the literature.

Case report: We report on a case of complete URSMS associated with Mayer-Rokitansky-Küster-Hauser syndrome and ovarian dysgenesis in a 46, XX newborn of 34 weeks' gestation. The most striking findings included abnormal development of the bladder and the lower portion of the vagina forming a single bulky multilocular mass, and of the gonads with an undifferentiated gonad and an ovary displaying teratomatous changes.

Conclusion: The association of URSMS with Mayer-Rokitansky-Küster-Hauser syndrome and ovarian dysgenesis do not appear fortuitous regarding the csommon mesodermal origin of the involved structures. This report illustrates the extreme phenotype of defect in mesodermal-derived structures development.

Keywords: Mayer-Rokitansky-Kuster-Hauser syndrome; Mesoderm; Ovary; Dysgenesis; Urorectal septum malformation sequence

\section{Introduction}

Urorectal septum malformation sequence (URSMS), also referred to as cloacal dysgenesis sequence, is a sporadic developmental disorder. It is related to the failure of division of the cloaca into anterior urogenital sinus and posterior anorectal canal by the urorectal septum at a very early stage of embryonic development (between weeks 4 and 6 of embryonic development) [1]. The embryonic mechanisms of the cloacal septation 


\section{Genomics \& Gene Therapy International Journal}

remain poorly understood. But, it is well established that the URSMS associated phenotype depends on the moment of arrest of the septation process. Considering the published cases, URSMS encompasses a broad spectrum of gastrointestinal and urogenital abnormalities [1-6]. The clinical phenotype ranges from a complete form that is usually lethal because of lung hypoplasia and renal failure, to a mild variant that is characterized by anteposed anus due to urorectal septum hypoplasia [1]. Here, we present a case of complete URSMS associated with Mayer-Rokitansky-Kuster-Hauser syndrome and ovarian dysgenesis in a female preterm newborn.

\section{Case report}

A 34-week-old neonate was born by caesarian section to a 25-year-old gravida 2, para 2 mother because of fetal distress of unknown cause.

The external genitalia were ambiguous, with pseudophalus and partially fused labia majora (Figure 1a). The perineum was smooth without patent urethral, vaginal and anal openings. X-ray showed asymmetry of the S4 and S5 vertebrae with agenesis of right pedicles and irregular, linear opacities in the right upper quadrant of the abdomen (Figure 1b). On internal examination, the lungs were slightly hypertrophic: right lung weighed 17 , $50 \mathrm{~g}$ (normal 15-15.8 g) and left 14, $76 \mathrm{~g}$ (normal 13.6$14.3 \mathrm{~g})$. There was esophageal atresia associated with distal tracheoesophageal fistula (type IV).

The small intestine showed multiple fibrinous adhesions which were related to prenatal meconium peritonitis (Figure 1c). The terminal portion of the colon was dilated and ended blindly on the wall of a markedly distended bladder (Figures 1c \& 1d). Bilateral ureteropyelohydronephrosis, more severe in the right side, was noted. The right kidney and adrenal gland were hypoplastic, weighing 8.21 g (normal 9.25-10 g) and 1.75 g (normal 3.15-3.4 g), respectively. On section, the megacystis was multilocular (Figure 1d), the renal cortices and medullae were thin and well demarcated and the renal calyces were severely dilated. Persistent omphalomesenteric duct and a single umbilical artery were observed. Two gonadal strips, measuring 7 (the right) and $17 \mathrm{~mm}$ (the left) along their major axis, have been identified on both sides of the bladder mass without identifiable female genital tract. On histological examination, the lungs revealed the presence of acellular eosinophilic hyaline membranes lining the alveolar ducts and numerous enlarged foamy alveolar macrophages.

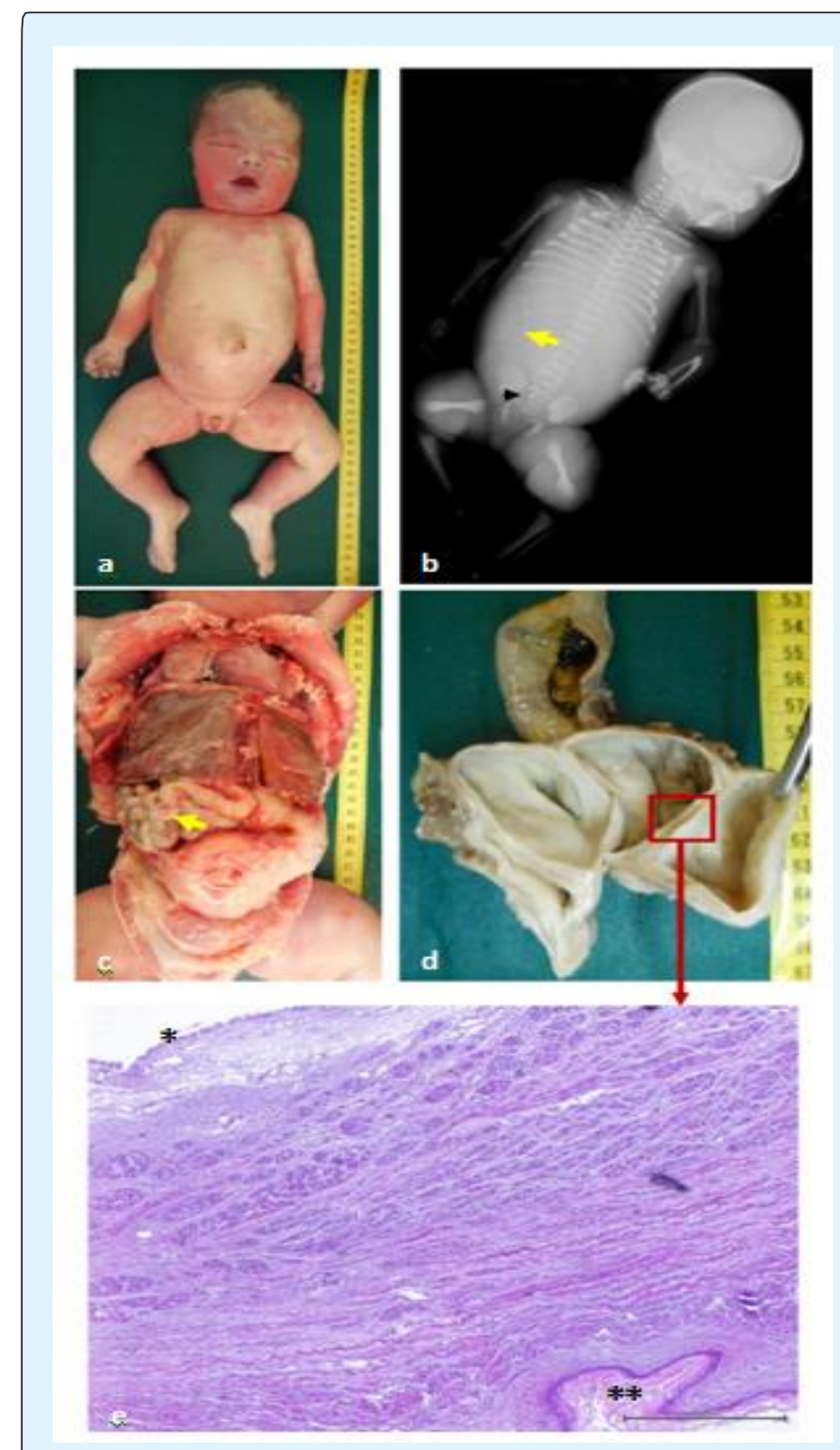

Figure 1: (a): Ambiguous genitalia with pseudophalus and partially fused labia majora. (b): Asymmetry of the S4 and S5 vertebrae with agenesis of right pedicles (black arrow) and presence of calcifications in the right upper quadrant of the abdomen (yellow arrow). (c): Megacystis with colovesical fistula. The small intestine showed multiple fibrinous adhesions (yellow arrow) (d): On section, the megacystis is multilocular. (e): Histology: the wall of the upper cavity draining the ureters was of histologic bladder structure, lined by transitional epithelium $\left(^{*}\right.$ ) with hypertrophic smooth muscle wall, while that of the lower blind cavity was of vaginal structure, lined by nonkeratinized malpighian epithelium $(* *)(H \& E, \times 40)$. 


\section{Genomics \& Gene Therapy International Journal}

The megacystis showed that the wall of the upper cavity draining the ureters was of histologic bladder structure, lined by a transitional epithelium with hypertrophic smooth muscle wall. The lower blind cavity corresponded to an atretic vagina with hydrocolpos; the wall was lined by nonkeratinized malpighian epithelium (Figure 1e). Histological study of the gonads showed a dysgenetic left ovary and an undifferentiated right gonad.

The dysgenetic ovary showed numerous primordial follicles in the stroma (Figures $2 a \& 2 b$ ).

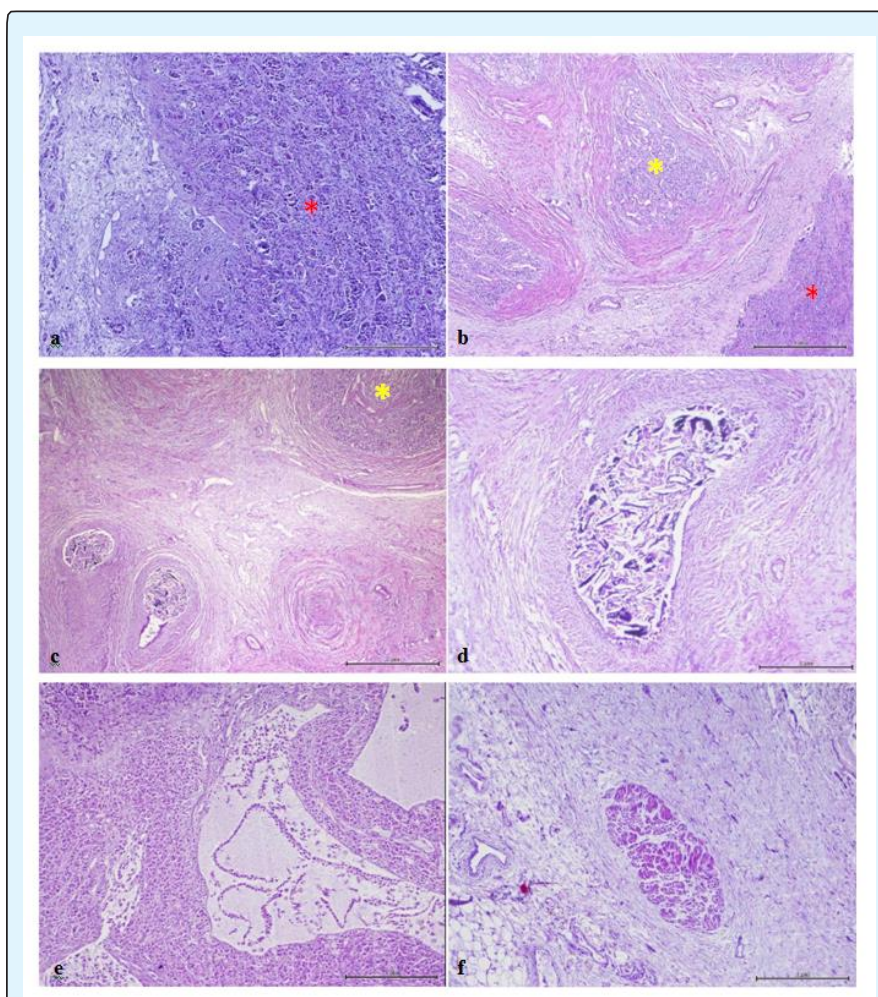

Figure 2: Left ovary: numerous primordial follicles in the stroma (a, b; red star), presence of fascicles of immaturelooking mesenchyme-like cells lined by concentric layers of smooth muscle cells (b, c ; yellow star), tubular structures lined by a low-prismatic epithelium and containing calcifications evoking embryonic vestiges of Wolff canal (c, d), adrenal cortex-like and cystic structures (e), and fascicles of smooth muscle cells in the areolar connective tissue (f). (H\&E; $a \times 100, b \times 40, c \times 40, d \times 100$, $\mathrm{e} \times 40, \mathrm{f} \times 20$ ).

The supporting stroma exhibited collections of ganglion cells and nerve fibers, adrenal cortex-like, fascicles of smooth muscle cells, cystic structures and tubular structures lined by a regular low-prismatic epithelium and containing calcifications, evoking embryonic remnants of Wolff canal (Figures $2 b$ \& 2 f). It also showed fascicles of immature-looking mesenchymelike cells lined by concentric layers of smooth muscle cells (Figures 2b \& 2c). The undifferentiated gonad was composed of haphazardly arranged fascicles of immaturelooking mesenchyme-like cells with areas of mature areolar and adipose connective tissues (Figure 3).

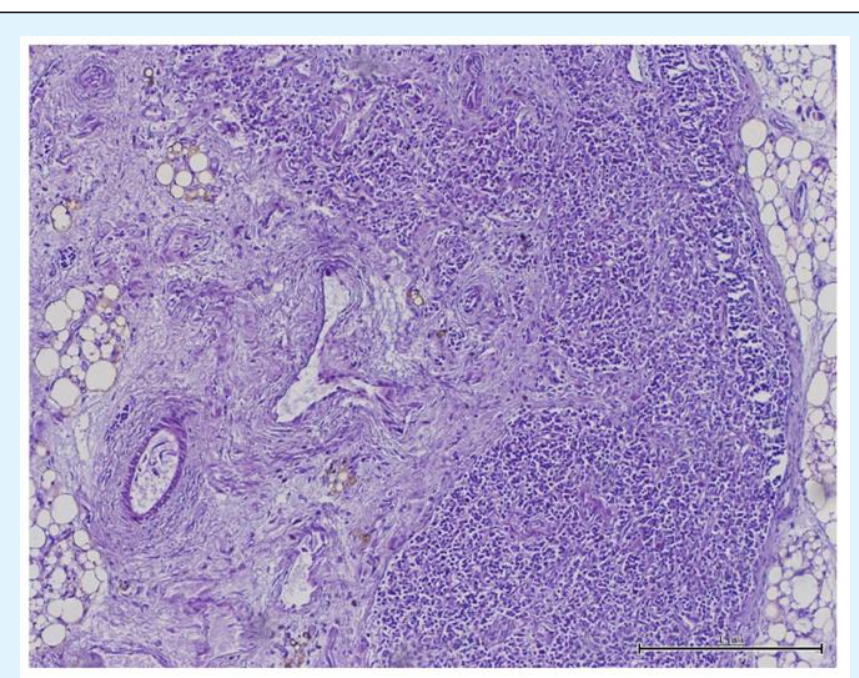

Figure 3: Right undifferentiated gonad: haphazardly arranged fascicles of immature-looking mesenchyme-like cells with areas of mature areolar and adipose connective tissues $(\mathrm{H} \& \mathrm{E} ; \times 40)$.

The placenta was normotrophic and didn't show significant histological lesions. Genetic analysis was not performed due to financial constraints.

\section{Discussion}

Our case report illustrates an unusual presentation of URSMS that is associated with both MRKH syndrome and ovarian dysgenesis. The common mesodermal origin of the three embryonic structures (urorectal septum, Mûllerian ducts and genital crests) allows stating that this association is probably not fortuitous but, rather, may represent the most extreme form of defect in embryonic mesoderm development. The complete URSMS is a congenital malformation that often results in prematurity and neonatal death because of hyaline membrane disease, similarly to our case $[1,4,5]$. The most striking abnormality detected by ultrasound and confirmed by autopsy in our case was the abdomino-pelvic mass which had an unusual appearance and size and was confused with megacystis. Thus, similar feature should alert the gynecologist to the possibility of URSMS. Other abnormalities may be detected by ultrasound including 


\section{Genomics \& Gene Therapy International Journal}

non-visualization of the anal sphincter complex, disorders of sex differentiation and meconial calcifications suggestive of uro-digestive fistula $[2,7,8]$.

The URSMS exhibits a wide spectrum of malformations. It encompasses constant abnormalities of the uro-genital and digestive tracts, usually associated with vesicovagino $\neg$ rectal fistulas [6]. In our case, two digestive atresias were observed: esophageal atresia associated with distal tracheoesophageal fistula and colo-rectal atresia without patent colovesical fistula. The latter resulted in intestinal perforation in utero and meconium peritonitis.

Pulmonary hypoplasia related to oligoamnios is a common, but inconstant finding. Polyhydramnios related to the esophageal atresia promoted lung growth in our case. Besides, the vertebral anomalies are frequently reported especially involving the sacrum similarly to our case [6]. The URSMS manifestations, described here, overlap other polymalformative syndromes, especially obstructive uropathy and VATER/VACTERL(S) association. Yet, uro-rectal malformations and abnormalities of sex differentiation allow distinguishing between these entities.

Our case presented with three characteristic features that have not been previously described in the literature. The first feature corresponded to the abnormal development of the bladder and the lower portion of the vagina forming a single bulky multilocular mass. Such malformation can be detected by ultrasound, but needs histology for definitive diagnosis. The second feature was the association of URSMS with MRKH syndrome and ovarian dysgenesis, which has not been reported yet in the English literature. MRKH syndrome is a congenital agenesis of the Mûllerian derivatives including fallopian tubes, uterus and two-thirds of the upper vagina. Abnormalities of the Mûllerian tract consisting of agenesis or malformation of the uterus and/or vagina are reported in most affected infants carrying URSMS without being able to specify whether it is a fortuitous association of malformative syndromes or a unique malformative sequence $[1-3,5]$. Besides, the association of MRKH syndrome with gonadal dysgenesis in 46 , XX patients is reported in the literature, though etiology of this association remains controversial [9]. The third feature was the abnormal development of the gonads. One gonad was of ovarian type as it contained follicles. But, it showed teratomatous changes, exhibiting multiple mature tissue types that included adrenal cortex-like, smooth muscle tissue, nervous tissue and cystic and tubular structures.
The other gonad was undifferentiated, didn't contain follicles and composed of areas of haphazardly arranged fascicules of mesenchyme-like cells and matures are olar and adipose connective tissues.

The etiopathogenic mechanism of URSMS is still obscure. The teratogen exposure during the early embryonic development may alter the mesoderm-specific gene expression preventing cloacal septation, as it is stated by Robinson and Tross [10]. In our case, no teratogenic factor was identified. Genetic mechanism is strongly involved though no gene has been identified yet. Familial occurrence suggests genetic etiology with a possible autosomal dominant mode of transmission $[7,11,12]$. It's noteworthy that both discordance and concordance in monozygotic twins are described [12]. Thus, multifactorial etiology is considered for this rare birth defect [12]. All reported children carrying this cloacal malformation, including our case, have normal karyotype. The only case of URSMS associated with chromosomal aberration (isochromosome 18q) is described by Chen et al. (1998) [13]. In our case, there was no family history of URSMS.

Recent studies state that the etiological mechanisms of MRKHS include single-gene mutations involving Wnt4, LHX1, HNF1B and TBX6, epigenetic changes and chromosomal translocations. Molecular investigation of this disorder is in progress [14]. Thus, the association of URSMS with MRKHS and ovarian dysgenesis does not appear to be fortuitous and would be linked to common molecular mechanisms involving genes that control the development of the mesodermal-derived structures.

\section{Conclusion}

We described an atypical presentation of complete URSMS which is associated with Mûllerian aplasia syndrome and ovarian dysgenesis in a 46, XX newborn. Recent advances in the molecular mechanisms involve defects in mesodermal gene expression that would otherwise explain the overlap of these congenital disorders.

Conflict of Interest: The authors declare that they have no conflict of interest.

\section{References}

1. Wheeler PG, Weaver DD, Obeime MO, Vance GH, Bull MJ, et al. (1997) Urorectal septum malformation sequence: report of thirteen additional cases and 


\section{Genomics \& Gene Therapy International Journal}

review of the literature. Am J Med Genet 73(4): 456462.

2. Liang X, Ioffe OB, Sun CC (1998) Cloacal dysgenesis sequence observations in four patients including three fetuses of second trimester gestation. Pediatr Dev Pathol 1(4): 281-288.

3. Wheeler PG, Weaver DD (2001) Partial urorectal septum malformation sequence: a report of 25 cases. Am J Med Genet 103(2): 99-105.

4. Bargaje A, Yerger JF, Khouzami A, Jones C (2008) Cloacal dysgenesis sequence. Ann Diagn Pathol 12(1): 62-66.

5. Jain D, Sharma MC, Kulkarni KK, Aggrawal S, Karak AK (2008) Urorectal septum malformation sequence: a report of seven cases. Congenit Anom (Kyoto) 48(4): 174-179.

6. Shah K, Nayak SS, Shukla A, Girisha KM (2016) Spectrum of urorectal septum malformation sequence. Congenit Anom (Kyoto) 56(3): 119-126.

7. Aggarwal, S, Phadke SR (2013) Recurrence of urorectal septum malformation sequence spectrum anomalies in siblings time to explore the genetics. Am J Med Genet A 161A (7): 1718-1721.

8. Pei Y, Wu Q, Liu Y, Sun L, Zhi W, et al. (2016) Prenatal sonographic diagnosis of urorectal septum malformation sequence and chromosomal microarray analysis: A case report and review of the literature. Medicine (Baltimore) 95(45): e5326.

9. Shah VN, Ganatra PJ, Parikh R, Kamdar P, Baxi S, et al. (2013) Coexistence of gonadal dysgenesis and MayerRokitansky-Kuster-Hauser syndrome in 46, XX female: A case report and review of literature. Indian J Endocrinol Metab 17(1): S274-S277.

10. Robinson HB, Tross JK (1984) Agenesis of the cloacal membrane. A probable teratogenic anomaly. Perspect Pediatr Pathol 8(1): 79-96.

11. Mills PL, Pergament E (1997) Urorectal septal defects in a female and her offspring. Am J Med Genet 70(3): 250-252.

12. Lubusky M, Prochazka M, Dhaifalah I, Halek J, Mickova I, et al. (2006) Concordant partial urorectal septum malformation sequence in monozygotic twins. Am J Med Genet A 140(24): 2828-2831.

13. Chen CP, Chern SR, Lee CC, Town DD (1998) Isochromosome $18 \mathrm{q}$ in a fetus with congenital megacystis, intra-uterine growth retardation and cloacal dysgenesis sequence. Prenat Diagn 18(10): 1068-1074.

14. Watanabe K, Kobayashi Y, Banno K, Matoba Y, Kunitomi H, et al. (2017) Recent advances in the molecular mechanisms of Mayer-Rokitansky-KüsterHauser syndrome. Biomed Rep 7(2): 123-127. 